\title{
A "Sinc-Galerkin" Method of Solution of Boundary Value Problems
}

\author{
By Frank Stenger**
}

\begin{abstract}
This paper illustrates the application of a "Sinc-Galerkin" method to the approximate solution of linear and nonlinear second order ordinary differential equations, and to the approximate solution of some linear elliptic and parabolic partial differential equations in the plane. The method is based on approximating functions and their derivatives by use of the Whittaker cardinal function. The DE is reduced to a system of algebraic equations via new accurate explicit approximations of the inner products, the evaluation of which does not require any numerical integration. Using $n$ function evaluations, the error in the final approximation to the solution of the $\mathrm{DE}$ is $O\left(e^{-c n^{1 / 2 d}}\right)$, where $c$ is independent of $n$, and $d$ denotes the dimension of the region on which the DE is defined. This rate of convergence is optimal in the class of $n$-point methods which assume that the solution is analytic in the interior of the interval, and which ignore possible singularities of the solution at the endpoints of the interval.
\end{abstract}

1. Introduction and Summary. The function $\operatorname{sinc}(x)$ is defined on the real line by

$$
\operatorname{sinc}(x)=\frac{\sin (\pi x)}{\pi x}
$$

The Whittaker cardinal function of an arbitrary function $f$ is defined for any $h>0$ by

$$
C(f, h, x)=\sum_{k=-\infty}^{\infty} f(k h) \operatorname{sinc}\left[\frac{x-k h}{h}\right], \quad h>0,
$$

whenever this series converges.

The approximation of $f$ using a finite number of terms of (1.2) has been extensively studied. The paper [8] contains a review of the properties of $C(f, h, x)$, which were discovered by E. T. Whittaker [16], J. M. Whittaker [17], Hartly [5], Nyquist [9] and Shannon [12]. In [13] new approximations are derived by means of $C(f, h, x)$, for interpolating, integrating and approximating the Fourier (over $(-\infty$, $\infty)$ only) and Hilbert transforms over $(-\infty, \infty),(0, \infty)$ and $(-1,1)$. In [7] the function $C(f, h, x)$ is used to obtain formulas for approximating the derivatives of functions over $(-\infty, \infty),(0, \infty)$ and $(-1,1)$.

In the present paper we use the results of [13] for solving second order

Received June 6, 1977.

AMS (MOS) subject classifications (1970). Primary 65N10, 65N30, 65N35; Secondary $65 \mathrm{~N} 15$.

* Research supported by NRC Grants \#A-3973, A-3990, and A-9239 at the University of British Columbia, and by U.S. Army Research Contract \#DAAG-29-76-G-0210.

** Current address: Department of Mathematics, University of Utah, Salt Lake City, Utah 84112. 
ordinary and partial differential equation boundary value problems. For purposes of explanation of the procedure, we consider the following situation. Let $d>0$, let $D_{d}$ denote the region $\{z=x+i y:|y|<d\}$ in the complex plane and let $\phi$ be a conformal map of a simply-connected domain $D$ onto $D_{d}$, such that $\phi(a)=-\infty$ and $\phi(b)=\infty$, where $a$ and $b(\neq a)$ are boundary points of $D$. Let $\psi$ denote the inverse of $\phi$, set $\Gamma=\psi((-\infty, \infty))$ and $x_{k}=\psi(k h), k=0 \pm 1, \pm 2, \ldots$, where $h>0$.

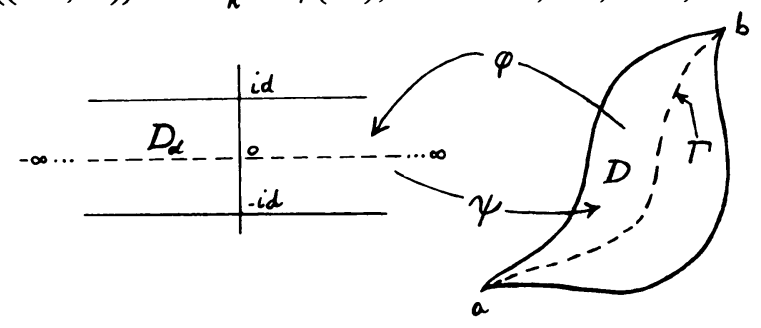

Figure 1.1. The domains $D$ and $D_{d}$

We seek an approximate solution of the linear boundary value problem

$$
\begin{gathered}
L(f)(x)=f^{\prime \prime}(x)+\mu(x) f^{\prime}(x)+\nu(x) f(x)-\sigma(x)=0, \quad x \in \Gamma, \\
f(a)=f(b)=0,
\end{gathered}
$$

on $\Gamma$. To this end, we assume that $\mu, \nu$ and $\sigma$ are analytic in $D$, and that (1.3) has a unique solution $f$ on $\Gamma$, which is analytic in $D$. We furthermore assume that

$$
\begin{gathered}
\int_{\partial D}\left|\frac{f^{\prime \prime}(z)}{\phi^{\prime}(z)} d z\right|<\infty ; \\
\int_{\partial D}\left|\frac{f^{\prime}(z) \mu(z)}{\phi^{\prime}(z)} d x\right|<\infty ; \\
\int_{\partial D}\left|\frac{f(z) \nu(z)}{\phi^{\prime}(z)} d z\right|<\infty ; \\
\int_{\partial D}\left|\frac{\sigma(z)}{\phi^{\prime}(z)} d z\right|<\infty ;
\end{gathered}
$$

and

$$
|f(x)| \leqslant C e^{-\alpha|\phi(x)|} \quad \text { on } \Gamma,
$$

where $C$ and $\alpha$ are positive constants, and where $\partial D$ denotes the boundary of $D$.

Notice that the above assumptions allow $f$ to have singularities at $a$ and $b$.

We approximate $f$ on $\Gamma$ by

$$
f(x) \cong f_{N}(x) \equiv \sum_{k=-N}^{N} f_{k} S(k, h) \circ \phi(x),
$$

where

$$
S(k, h)(x)=\frac{\sin \left[\frac{\pi}{h}(x-k h)\right]}{\frac{\pi}{h}(x-k h)}
$$


The general Galerkin method enables us to determine the $f_{k} \cong f\left(x_{k}\right)$ by solving the linear system of equations $\left(L f_{N}, S(k, h) \circ \phi\right)=0, k=-N,-N+1, \ldots, N$. We choose instead to obtain an approximate solution of the system

$$
(L(f), S(k, h) \circ \phi)=0, \quad k=-N,-N+1, \ldots, N,
$$

where the inner product in (1.11) is defined by

$$
(u, v)=\int_{\Gamma} g(x) u(x) \overline{v(x)} d x,
$$

where $g$ plays the role of a weight function. For the case of second order problems, it is convenient to take

$$
g(x)=\frac{1}{\phi^{\prime}(x)}
$$

Let us use the notation

$$
\delta_{j k}^{(0)}=\left\{\begin{array}{ll}
1 & \text { if } j=k, \\
0 & \text { if } j \neq k,
\end{array} \quad \delta_{j k}^{(1)}= \begin{cases}0 & \text { if } j=k, \\
\frac{(-1)^{k-j}}{k-j}, & \text { if } j \neq k,\end{cases}\right.
$$

$$
\delta_{j k}^{(2)}= \begin{cases}-\frac{\pi^{2}}{3} & \text { if } j=k, \\ \frac{-2(-1)^{k-j}}{(k-j)^{2}} & \text { if } j \neq k .\end{cases}
$$

With this notation, we obtain the following approximate explicit expressions for the inner products in (1.11):

$$
\begin{aligned}
& \int_{\Gamma} g(x) \sigma(x) S(k, h) \circ \phi(x) d x \cong h \frac{g_{k} \sigma_{k}}{\phi_{k}^{\prime}} ; \\
& \int_{\Gamma} g(x) \mu(x) f(x) S(k, h) \circ \phi(x) d x \cong h \frac{g_{k} \mu_{k}}{\phi_{k}^{\prime}} f_{k} ; \\
& \int_{\Gamma} g(x) \nu(x) f^{\prime}(x) S(k, h) \circ \phi(x) d x \\
& \cong h \frac{g_{k} \nu_{k}}{\phi_{k}^{\prime}} f_{k}^{\prime} \cong-h \sum_{j=-N}^{N} f_{j}\left\{\frac{(\nu g)_{j}^{\prime}}{\phi_{j}^{\prime}} \delta_{k j}^{(0)}+(\nu g)_{j} \frac{\delta_{k j}^{(1)}}{h}\right\} ; \\
& \int_{\Gamma} g(x) f^{\prime \prime}(x) S(k, h) \circ \phi(x) d x \cong h \frac{g_{k} f_{k}^{\prime \prime}}{\phi_{k}^{\prime}} \\
& \cong h \sum_{j=-N}^{N} f_{j}\left\{\frac{g_{j}^{\prime \prime}}{\phi_{j}^{\prime}} \delta_{k j}^{(0)}+\left(2 g_{j}^{\prime}+g_{j} \phi_{j}^{\prime \prime} / \phi_{j}^{\prime}\right) \frac{\delta_{k j}^{(1)}}{h}+g_{j} \phi_{j}^{\prime} \frac{\delta_{k j}^{(2)}}{h^{2}}\right\},
\end{aligned}
$$


where $g_{k}=g\left(x_{k}\right)$, etc. These explicit expressions make "collocation" and "Galerkin" synonymous for this method. The choice $h=(\pi d / \alpha N)^{1 / 2}$ and $g(x)=1 / \phi^{\prime}(x)$ yields $O\left(N^{1 / 2} e^{-(\pi d \alpha N)^{1 / 2}}\right)$ accuracy for each of the approximations in (1.15).

Using (1.15) in (1.11), we obtain a linear system of equations for $2 N+1$ numbers $f_{k}$. The dominant matrix of the system is $\left[\delta_{k j}^{(2)}\right]$ (see Eq. (2.47)). Contrary to the case of finite difference or finite element methods, which lead to sparse matrices, the matrix $\left[\delta_{k j}^{(2)}\right]$ is a well-conditioned full symmetric negative definite matrix, with condition number less than $(N+1)^{2}$.

The resulting approximation $f_{N}$ in (1.9) satisfies

$$
\left|f(x)-f_{N}(x)\right| \leqslant C^{\prime} N^{3 / 2} e^{-(\pi d \alpha N)^{1 / 2}}
$$

for all $x \in \Gamma$. While $n$-point finite difference or finite element methods converge at the rate $O\left(n^{-P}\right)$, where $P$ is usually 0 or 1 , the rate of convergence (1.16) cannot be improved [15]. That is, there is no basis $\left\{\psi_{k}^{(N)}\right\}_{k=-N}^{N}$ such that ${ }^{1} \phi_{N}(x)=$ $\Sigma_{k=-N}^{N} c_{k} \psi_{k}^{(N)}(x)$ converges to $f$ faster than the rate (1.16) for all problems of the type (1.3) satisfying the above analyticity assumptions and the conditions (1.4)-(1.8).

For example, if $a=0, b=1$ in (1.3), it is convenient to take for $D$ the region

$$
D=\{z=x+i y:|\arg [z /(1-z)]|<d\}
$$

(see Figure 2.1). Then $\phi(z)=\log [z /(1-z)], \phi^{\prime}(z)=z^{-1}(1-z)^{-1}$ and $\psi(w)=$ $1 / 2+1 / 2 \tanh (w / 2)$. In this case $x_{k}=1 / 2+1 / 2 \tanh (k h / 2)$, and $\Gamma=\psi((-\infty, \infty))=(0,1)$. We could then use the above procedure to solve problems having regular singular points at 0 and 1 , such as

$$
f^{\prime \prime}-\frac{\cos 2 \pi x}{x(1-x)} f^{\prime}+\frac{2 x^{2}+1}{x^{2}(1-x)^{2}} f-x^{\alpha-1}(1-x)^{\alpha-2}=0, \quad f(0)=f(1)=0
$$

or singular perturbation problems, such as

$$
\epsilon f^{\prime \prime}-\frac{3}{1-x} f=\frac{x^{3} e^{-x}}{1-x}, \quad f(0)=f(1)=0 .
$$

For the problem (1.18) and (1.19) the condition (1.8) reduces to $|f(x)| \leqslant C x^{\alpha}(1-x)^{\alpha}$ on $(0,1)$. If $\epsilon$ is small in $(1.19)$, then it may be necessary to take $\alpha$ small in order to satisfy this condition, resulting in a larger error (see (1.16)).

Nonlinear equations can similarly be handled. For example, a term such as $F\left(x, f(x), f^{\prime}(x)\right)$ appearing in the equation $L(f)=f^{\prime \prime}+F\left(x, f, f^{\prime}\right)=0$ is handled in (1.11) via the formula

$$
\int_{\Gamma} g(x) F\left(x, f(x), f^{\prime}(x)\right) S(k, h) \circ \phi(x) d x \cong h F\left(x_{k}, f_{k}, f_{k}^{\prime}\right) \frac{g_{k}}{\phi_{k}^{\prime}},
$$

and the third of (1.15) may now be used to replace $f_{k}^{\prime}$ by a linear combination of $f_{-N}, f_{-N+1}, \ldots, f_{N}$.

${ }^{1}$ We mention that classical finite difference methods or finite element methods are based on achieving (locally or globally) exactness for polynomials of certain degree. Such a method can never converge faster than $O\left(n^{-P}\right)$ in the presence of singularities. The basis functions of the present method are not polynomials; rather, they are entire functions composed with conformal maps. 
In Section 2 of the paper we derive the approximation formulas (1.15), along with error bounds, we give a brief description of the matrices $\left[\delta_{j k}^{(1)}\right]$ and $\left[\delta_{j k}^{(2)}\right]$; and we give explicit derivations of $(1.15)$ for the special intervals $(0,1),(-1,1),(0, \infty)$ and $(-\infty, \infty)$. In Section 3 we illustrate the application of the previously derived formulas to the approximate solution of the simple "model" problems $f^{\prime \prime}=-2, f$ " $=f$ $f^{3} / x^{2}, u_{t}=u_{x x}$ and $u_{x x}+u_{y y}=1$, with appropriate boundary conditions. Typically, a matrix of order 33 yields $4-5$ decimal accuracy in all of these cases. In Section 4 we carry out an error analysis, proving the $O\left(e^{-c n^{1 / 2}}\right)$ rate of convergence referred to above.

The approximate methods of [7], [13] have previously been effectively applied to the approximate solution of integral equations via Galerkin-type methods in [2], [10], [11]. In [6] an effective Galerkin-type method is derived in [13] to obtain an approximate solution to the problem $f^{\prime \prime}=f-f^{3} / x^{2}, f(0)=f(\infty)=0$ via the minimization of a certain nonlinear functional. In all of these cases the error of an approximate solution converges at the optimal rate, $O\left(e^{-c n^{1 / 2}}\right)$.

2. Preliminaries and Fundamentals. In this section we shall recall some known properties [8] and derive some new properties of Whittaker's cardinal function, which we shall require in this paper.

Definition 2.1. Let $R$ denote the real line, $C$ the complex plane, and let $B(h)$ denote the family of all functions defined on $C$ that are entire, such that $f \in L^{2}(R)$ and such that

$$
|f(z)| \leqslant C e^{\pi|y| / h}, \quad z=x+i y \in C,
$$

for some constant $C$. Set

$$
S(j, h)(x)=\operatorname{sinc}\left[\frac{x-j h}{h}\right]
$$

and

$$
\delta_{j k}^{(n)}=S^{(n)}(j, 1)(k)=\left.\left(\frac{d}{d x}\right)^{n} S(j, 1)(x)\right|_{x=k} .
$$

In particular, we have

$$
\begin{aligned}
& \delta_{j k}^{(0)}= \begin{cases}1 \quad \text { if } j=k, \\
0 & \text { if } j \neq k,\end{cases} \\
& \delta_{j k}^{(1)}= \begin{cases}0 & \text { if } j=k, \\
\frac{(-1)^{k-j}}{k-j} & \text { if } j \neq k,\end{cases} \\
& \delta_{j k}^{(2)}= \begin{cases}-\frac{\pi^{2}}{3} & \text { if } j=k, \\
\frac{-2(-1)^{k-j}}{(k-j)^{2}} & \text { if } j \neq k .\end{cases}
\end{aligned}
$$


THEOREM $2.2[8]$. Let $f \in B(h)$. Then $f(z)=C(f, h, z)$. Moreover,

$$
\begin{gathered}
f(z)=\int_{-\pi / h}^{\pi / h} g(t) e^{i z t} d t \quad \text { for some } g \in L^{2}\left(-\frac{\pi}{h}, \frac{\pi}{h}\right) \\
f(z)=\frac{1}{h} \int_{R} \operatorname{sinc}\left[\frac{z-t}{h}\right] f(t) d t \\
\int_{R}|f(x)|^{2} d x=h \sum_{k=-\infty}^{\infty}|f(k h)|^{2}
\end{gathered}
$$

and the sequence $\left\{h^{-1 / 2} S(k, h)\right\}_{k=-\infty}^{\infty}$ is, therefore, a complete orthonormal sequence in $B(h)$;

$$
f \in B(h) \Rightarrow f^{\prime} \in B(h)
$$

THEOREM 2.3. Let $\delta_{j k}^{(n)}$ be defined as in (2.3). Then

$$
\int_{R}\left\{\operatorname{sinc}\left[\frac{x-j h}{h}\right]\right\}^{(n)} \operatorname{sinc}\left[\frac{x-k h}{h}\right] d x=h^{1-n} \delta_{j k}^{(n)}, \quad n=0,1,2, \ldots
$$

Proof. Let us set

$$
f(t)=S(j, h)(t)
$$

and let us note that $f \in B(h)$. By Eq. (2.8) it thus follows that $f^{(n)} \in B(h), n=$ $0,1,2, \ldots$ Equation (2.9) thus follows by taking $f=S(j, h)^{(n)}$ in (2.6), and noting by (2.3) that

$$
S^{(n)}(j, h)(k h)=h^{-n} \delta_{j k}^{(n)}
$$

Definition 2.4. Let $d>0$, and let $B\left(D_{d}^{\prime}\right)$ denote the family of all functions $f$ that are analytic in

$$
D_{d}^{\prime}=\{z=x+i y:|y|<d\}
$$

such that

$$
\int_{-d}^{d}|f(x+i y)| d y \rightarrow 0 \text { as } x \rightarrow \pm \infty
$$

and such that $N\left(f, D_{d}^{\prime}\right)<\infty$, where

$$
N\left(f, D_{d}^{\prime}\right)=\lim _{y \rightarrow d^{-}}\left\{\int_{R}|f(x+i y)| d x+\int_{R}|f(x-i y)| d x\right\}
$$

ThEOREM $2.5[13]$. Let $h$ and $d$ be positive, let $f \in B\left(D_{d}^{\prime}\right)$, and let $\epsilon(f)$ be defined by

$$
\epsilon(f)(x)=f(x)-C(f, h, x), \quad x \in R
$$


Then,

$$
\epsilon(f)(x)=\frac{\sin (\pi x / h)}{2 \pi i} \int_{R}\left[\frac{f\left(t-i d^{-}\right)}{(t-x-i d) \sin [(t-i d) \pi / h}\right]
$$

$$
\left.-\frac{f\left(t+i d^{-}\right)}{(t-x+i d) \sin [(t+i d) \pi / h]}\right] d t .
$$

Moreover,

$$
\|\epsilon(f)\|_{\infty}=\sup _{x \in R}|\epsilon(f)(x)| \leqslant \frac{N\left(f, D^{\prime}\right)}{2 \pi d \sinh (\pi d / h)} .
$$

Definition 2.6. Let $D$ be a simply-connected domain in the complex plane $C$, and let $D_{d}^{\prime}$ be defined as in (2.12). Let $\phi$ be a conformal map of $D$ onto $D_{d}^{\prime}$, and let $\psi=\phi^{-1}$ denote the inverse map. Let $a=\psi(-\infty)$ and $b=\psi(\infty) \neq a$ be boundary points of $D$, and let us take

$$
\Gamma=\{w \in D: w=\psi(x),-\infty \leqslant x \leqslant \infty\} .
$$

Let $B(D)$ denote the family of all functions that are analytic in $D$, such that for $u$ real

$$
\int_{\psi(L+u)}|f(z) d z| \rightarrow 0 \text { as } u \rightarrow \pm \infty
$$

where

$$
L=\{i y:-d \leqslant y \leqslant d\}
$$

and such that

$$
N(f, D)=\liminf _{C \rightarrow \partial D, C \subset D} \int_{C}|f(z) d z|<\infty .
$$

(Note that if $f \in B(D)$, then $f \circ \psi \in B\left(D_{d}^{\prime}\right)$.) Set

$$
x_{k}=\psi(k h), \quad k=0, \pm 1, \pm 2, \ldots,
$$

and let $g$ be a function which is analytic in $D$, which plays the role of a weight function in the inner products, and whose properties we shall determine in the sequel. Finally, we set

$$
S_{j}(z)=g(z) \operatorname{sinc}\left[\frac{\phi(z)-j h}{h}\right]=g(z) S(j, h) \circ \phi(z) .
$$

The following result was established in [7] .

THEOREM 2.7. Let $m$ be a nonnegative integer, and let $f \phi^{\prime} / g \in B(D)$. Let there exist positive constants $\alpha, C_{0}$ depending only on $m, d$ and $g, C_{1}$ depending only on $m$ and $g$, and $C_{2}$ depending only on $m, g$ and $f$, such that

$$
\begin{aligned}
& \left|\frac{f(x)}{g(x)}\right| \leqslant C_{2} e^{-\alpha|\phi(x)|} \quad \text { for all } x \in \Gamma, \\
& \left.\begin{array}{l}
\mid\left(\frac{d}{d x}\right)^{n} S_{k}(x)\left[\leqslant C_{1} h^{-n} \quad \text { for all } x \in \Gamma\right. \\
\left.\frac{(x) / h]}{(x)}\right\} \mid \leqslant C_{0} h^{-n} \quad \text { for all } x \in \Gamma, z \in \partial D
\end{array}\right\} \\
& n=0,1, \ldots, m \text {. }
\end{aligned}
$$


Then there exists a constant $K$ depending only on $m, d, \alpha, g$ and $f$ such that if $h=$ $[\pi d /(\alpha N)]^{1 / 2}$, then

$$
\left|f^{(n)}(x)-\sum_{j=-N}^{N} \frac{f\left(x_{j}\right)}{g\left(x_{j}\right)} S_{j}^{(n)}(x)\right| \leqslant K N^{(n+1) / 2} \exp \left[-(\pi d \alpha N)^{1 / 2}\right]
$$

for all $x \in \Gamma$, and for $n=0,1, \ldots, m$.

THEOREM $2.8[13]$. If $f \in B(D)$, then the identity ${ }^{2}$

$$
\begin{aligned}
\frac{f(x)}{\phi^{\prime}(x)}-\sum_{j=-\infty}^{\infty} \frac{f\left(x_{j}\right)}{\phi^{\prime}\left(x_{j}\right)} S(j, h) \circ \phi(x) \\
\quad=\frac{\sin [\pi \phi(x) / h]}{2 \pi i} \int_{\partial D} \frac{f(z) d z}{[\phi(z)-\phi(x)] \sin [\pi \phi(z) / h]}
\end{aligned}
$$

is valid for all $x \in \Gamma$. Moreover,

$$
\int_{\Gamma} f(x) d x-h \sum_{j=-\infty}^{\infty} \frac{f\left(x_{j}\right)}{\phi^{\prime}\left(x_{j}\right)}=\frac{1}{2} \int_{\partial D} \frac{\exp \left[\frac{i \pi \phi(z)}{h} \operatorname{sgn} \operatorname{Im} \phi(z)\right]}{\sin \left[\frac{\pi}{h} \phi(z)\right]} f(z) d z
$$

The results of this theorem may be conveniently combined with those of the formulas obtained above, to yield explicit approximate expressions for inner products. The results of the following lemma are useful for bounding the error of these approximate expressions.

LEMma 2.9. If $|\operatorname{Im} z|=d>0$ and if $k$ is an integer, then

$$
\frac{1}{2}\left|\frac{\operatorname{sinc}[(z-k h) / h]}{\sin (\pi z / h)}\right| \leqslant C_{1}(h, d) \equiv \frac{h}{2 \pi d} ;
$$

$$
\begin{gathered}
\frac{1}{2}\left|\frac{(d / d z)\{\operatorname{sinc}[(z-k h) / h]\}}{\sin (\pi z / h)}\right| \leqslant C_{2}(h, d) \equiv \frac{d+(h / \pi) \tanh (\pi d / h)}{2 d^{2} \tanh (\pi d / h)} ; \\
\frac{1}{2}\left|\frac{\frac{-d^{2}}{d z^{2}}\{\operatorname{sinc}[(z-k h) / h]\}}{\sin (\pi / h)}\right| \\
\leqslant C_{3}(h, d) \equiv \frac{\left[(2 h / \pi)+\pi^{2} d / h\right] d \tanh (\pi d / h)+2 d}{2 d^{3} \tanh (\pi d / h)} ; \\
|\sin (\pi z / h)| \geqslant \sinh (\pi d / h), \quad|\cos (\pi z / h)| \leqslant \cosh (\pi d / h) .
\end{gathered}
$$

Proof. We shall only prove $(2.31)$, since the proofs of the remaining cases are similar, and we omit them. We have

$$
w=\frac{d}{d z}\{S(k, h)(z)\}=\frac{\cos [\pi(z-k h) / h]}{z-k h}-\frac{h}{\pi} \frac{\sin [\pi(z-k h) / h]}{(z-k h)^{2}} .
$$

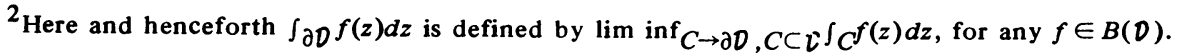


Now if $|\operatorname{Im} z|=d$, then $|z-k h| \geqslant d,|\cos [\pi(z-k h)]| \leqslant \cosh (\pi d / h)$ and $|\sin (\pi z / h)| \geqslant$ $\sinh (\pi d / h)$; hence

$$
\left|\frac{w}{\sin (\pi z / h)}\right| \leqslant \frac{1}{d \tanh (\pi d / h)}+\frac{h}{\pi d^{2}}=C_{2}(h, d)
$$

TheOREM 2.10. Let $\delta_{j k}^{(n)}$ be defined as in (2.3) and (2.4), let $C_{j}(h, d)$ be defined as in Lemma 2.9, let $x_{k}$ be defined as in (2.22), $S_{k}$ as in (2.23), set $F_{k}=$ $F\left(x_{k}\right)$ for an arbitrary function $F$, and let $r$ and $f$ be functions which are analytic in $D$.

(a) Let $r g \in B(D)$. Then

$$
\left|\int_{\Gamma} r(x) f(x) S_{k}(x) d x-h \frac{f_{k} r_{k} g_{k}}{\phi_{k}^{\prime}}\right| \leqslant C_{1}(h, d) N(r f g, D) e^{-\pi d / h} .
$$

(b) Let $[r f g / \phi](x) \rightarrow 0$ as $x \rightarrow a$ and as $x \rightarrow b$ along $\Gamma$, and let $(r g)^{\prime} f$ and $r g \phi^{\prime} f \in B(D)$. Then

$$
\begin{gathered}
\left|\int_{\Gamma} r(x) f^{\prime}(x) S_{k}(x) d x+h \sum_{j=-\infty}^{\infty} f_{j}\left\{\frac{(r g)_{j}^{\prime}}{\phi_{j}^{\prime}} \delta_{k j}^{(0)}+(r g)_{j} \frac{\delta_{k j}^{(1)}}{h}\right\}\right| \\
\leqslant\left[C_{1}(h, d) N\left(f(r g)^{\prime}, D\right)+C_{2}(h, d) N\left(f r g \phi^{\prime}, D\right)\right] e^{-\pi d / h}
\end{gathered}
$$

(c) Let $\left[\mathrm{frg}^{\prime} / \phi\right](x),\left[f r g \phi^{\prime} / \phi\right](x)$ and $\left[f^{\prime} r g / \phi\right](x) \rightarrow 0$ as $x \rightarrow a$ and as $x \rightarrow b$ along $\Gamma$, and let $f(r g)^{\prime \prime}, f\left[2(r g)^{\prime} \phi^{\prime}+r g \phi^{\prime \prime}\right]$ and $f r g\left(\phi^{\prime}\right)^{2} \in B(D)$. Then

$$
\begin{aligned}
& \mid \int_{\Gamma} r(x) f^{\prime \prime}(x) S_{k}(x) d x \\
& \quad-h \sum_{j=-\infty}^{\infty} f_{j}\left\{\frac{(r g)_{j}^{\prime \prime}}{\phi_{j}^{\prime}} \delta_{k j}^{(0)}+\frac{\left[2(r g)_{j}^{\prime} \phi_{j}^{\prime}+(r g)_{j} \phi_{j}^{\prime \prime}\right]}{\phi_{j}^{\prime}} \frac{\delta_{k j}^{(1)}}{h}+(r g)_{j} \phi_{j}^{\prime} \frac{\delta_{k j}^{(2)}}{h^{2}}\right\} \mid \\
& \leqslant\left[C_{1}(h, d) N\left(f(r g)^{\prime \prime}, D\right)+C_{2}(h, d) N\left(f\left\{2(r g)^{\prime} \phi^{\prime}+r g \phi^{\prime \prime}\right\}, D\right)\right. \\
& \left.+C_{3}(h, d) N\left(f r g\left(\phi^{\prime}\right)^{2}, D\right)\right] \cdot e^{-\pi d / h} .
\end{aligned}
$$

Proof. We shall only prove the (b)-part of Theorem 2.9, since the proofs of the (a)- and (c)-parts are similar.

We find, upon integration by parts, that

$$
\begin{aligned}
\int_{\Gamma} r(x) f^{\prime}(x) S_{k}(x) d x= & \left.r(x) f(x) S_{k}(x)\right|_{a} ^{b} \\
& -\int_{\Gamma} f(x)\left[r(x) S_{k}^{\prime}(x)+r^{\prime}(x) S_{k}(x)\right] d x
\end{aligned}
$$

The first term on the right-hand side vanishes, by assumption of the (b)-part of the 
theorem, while by expansion of the second part of (2.37), we have

$$
\int_{\Gamma} r(x) f^{\prime}(x) S_{k}(x) d x
$$

$$
=-\int_{\Gamma} f(x)\left[(r g)^{\prime}(x) S(k, h) \circ \phi(x)+\left(r g \phi^{\prime}\right)(x) S^{\prime}(k, h) \circ \phi(x)\right] d x .
$$

Hence by replacing $f$ in (2.29) by the integrand on the right-hand side of (2.38), and noting that if $z \in \partial D$, then $|\operatorname{Im} \phi(z)|=d$ and

$$
\left|\exp \left[\frac{i \pi}{h} \phi(z) \operatorname{sgn} \operatorname{Im} \phi(z)\right]\right|=e^{-\pi d / h}
$$

we find by (2.29), Lemma 2.9 and Theorem 2.3 , that

$$
\begin{aligned}
& \left|\int_{\Gamma} r(x) f^{\prime}(x) S_{k}(x) d x+h \sum_{j=-\infty}^{\infty} f_{j}\left\{\frac{(r g)_{j}^{\prime}}{\phi_{j}^{\prime}} \delta_{k j}^{(0)}+(r g)_{j} \frac{\delta_{k j}^{(1)}}{h}\right\}\right| \\
& \leqslant e^{-\pi d / h} \int_{\partial D}\left[C_{1}(h, d)\left|\left[f(r g)^{\prime}\right](z)\right|+C_{2}(h, d)\left|\left(f r g \phi^{\prime}\right)(z)\right|\right]|d z|,
\end{aligned}
$$

which is just (2.35).

THEOREM 2.11. Let $N$ be a positive integer, $\alpha$ a positive constant, and take $h=[\pi d /(\alpha N)]^{1 / 2}$.

(a) Under the assumptions of Theorem 2.9(a),

$$
\left|\int_{\Gamma} r(x) f(x) S_{k}(x) d x-h \frac{f_{k} r_{k} g_{k}}{\phi_{k}^{\prime}}\right| \leqslant \frac{K_{1}}{N^{1 / 2}} e^{-(\pi d \alpha N)^{1 / 2}},
$$

where $K_{1}$ depends only on $f, r, g, d$ and $\alpha$;

(b) If $|[r f g](x)| \leqslant K_{2}^{\prime} \exp [-\alpha|\phi(x)|]$ on $\Gamma$, then under the assumptions of Theorem 2.9(b),

$$
\begin{gathered}
\iint_{\Gamma} r(x) f^{\prime}(x) S_{k}(x) d x+h \sum_{j=-N}^{N} f_{j}\left\{\frac{(r g)_{j}^{\prime}}{\phi_{j}^{\prime}} \delta_{k j}^{(0)}+(r g)_{j} \frac{\delta_{k j}^{(1)}}{h}\right\} \mid \\
\leqslant K_{2} e^{-(\pi d \alpha N)^{1 / 2}}, \quad k=-N,-N+1, \ldots, N,
\end{gathered}
$$

where $K_{2}$ depends only on $f, r, g, d$ and $\alpha$;

(c) If $\left[f\left\{2(r g)^{\prime}+r g \phi^{\prime \prime} / \phi^{\prime}\right\}\right](x)$ and $\left[r g f \phi^{\prime}\right](x)$ are bounded by $K_{3}^{\prime} \exp [-\alpha|\phi(x)|]$ on $\Gamma$, then under the assumptions of Theorem 2.9(c),

$$
\iint_{\Gamma} r(x) f^{\prime \prime}(x) S_{k}(x) d x
$$

$$
\begin{aligned}
& -h \sum_{j=-N}^{\infty} f_{j}\left\{\frac{(r g)_{j}^{\prime \prime}}{\phi_{j}^{\prime}} \delta_{k j}^{(0)}+\frac{\left[2(r g)_{j}^{\prime} \phi_{j}+(r g)_{j} \phi_{j}^{\prime \prime}\right]}{\phi_{j}^{\prime}} \frac{\delta_{k j}^{(1)}}{h}+(r g)_{j} \phi_{j}^{\prime} \frac{\delta_{k j}^{(2)}}{h^{2}}\right\} \\
& \leqslant K_{3} N^{1 / 2} e^{-(\pi d \alpha N)^{1 / 2}}, \quad k=-N,-N+1, \ldots, N,
\end{aligned}
$$

where $K_{3}$ depends only on $f, r, g, d$ and $\alpha$. 
Proof. The proof is similar to that of Theorem 8.1 of [13], and we omit it.

The results of Theorem 2.10 are especially suited to the solution of linear differential equations via a Galerkin method, for which the functions $\left\{S_{k} / g\right\}$ are the approximating basis functions. We remark that we could have obtained alternate expressions of $\int_{\Gamma} r(x) f^{(n)}(x) S_{k}(x) d x$, by combining Eqs. (2.29) and (2.27), i.e., if $r g f^{(n)} \in B(D)$, then by Eq. $(2.29)$

$$
\begin{gathered}
\left|\int_{\Gamma} r(x) f^{(n)}(x) S_{k}(x) d x-\frac{h r_{k} g_{k} f^{(n)}\left(x_{k}\right)}{\phi_{k}^{\prime}}\right| \\
\leqslant C_{1}(h, d) N\left(r g f^{(n)}, D\right) e^{-\pi d / h} ;
\end{gathered}
$$

and we could now use (2.29) to approximate $f^{(n)}\left(x_{k}\right)$ on $\Gamma$. However, the resulting expressions are usually not as accurate as those of Theorem 2.10 . Nevertheless the pair of equations (2.27) and (2.29) do form a powerful combination for purposes of solving nonlinear equations. For example, if $g G \in B(\mathcal{D})$, where $G=G\left(x, f(x), f^{\prime}(x)\right)$, then

$$
\begin{aligned}
& \left|\int_{\Gamma} G\left(x, f(x), f^{\prime}(x)\right) S_{k}(x) d x-h \frac{G\left(x_{k}, f\left(x_{k}\right), f^{\prime}\left(x_{k}\right)\right)}{\phi^{\prime}\left(x_{k}\right)} g\left(x_{k}\right)\right| \\
& \leqslant C_{1}(h, d) N(G, D) e^{-\pi d / h}
\end{aligned}
$$

if the conditions of Theorem 2.7 are satisfied for $m=1$, we may now replace $f^{\prime}\left(x_{k}\right)$ in (2.43) by the approximation

$$
f^{\prime}\left(x_{k}\right) \cong \sum_{j=-N}^{N} \frac{f_{j}}{g_{j}}\left[g_{j}^{\prime} \delta_{j k}^{(0)}+g_{j} \phi_{j}^{\prime} \frac{\delta_{j k}^{(1)}}{h}\right]
$$

given by Eq. (2.27).

The approximating expressions of Theorem 2.10 may be more compactly expressed by means of matrices. To this end, let $m=2 N+1$, and let $\mathbf{S}_{m}$ and $\mathbf{f}_{m}$ be column vectors defined by

$$
\mathbf{S}_{m}(x)=\left(\begin{array}{c}
S_{-N}(x) \\
S_{-N+1}(x) \\
\cdot \\
\cdot \\
\cdot \\
S_{N}(x)
\end{array}\right), \quad \mathbf{f}_{m}=\left(\begin{array}{c}
f_{-N} \\
f_{-N+1} \\
\cdot \\
\cdot \\
\cdot \\
f_{N}
\end{array}\right) .
$$

Corresponding to a function $u=u(x)$, let $\mathbf{A}_{m}(u)$ denote a diagonal matrix, whose diagonal elements are $u\left(x_{-N}\right), u\left(x_{-N+1}\right), \ldots, u\left(x_{N}\right)$ and whose off-diagonal elements are zero. Let $\mathbf{I}_{m}^{(1)}$ and $\mathbf{I}_{m}^{(2)}$ denote the matrices 


$$
I_{m}^{(1)}=\left[\begin{array}{cccccc}
0 & -1 & \frac{1}{2} & -\frac{1}{3} & \cdots & \frac{1}{2 N} \\
1 & 0 & -1 & -\frac{1}{2} & \cdots & -\frac{1}{2 N-1} \\
-\frac{1}{2} & 1 & 0 & \cdots & & \frac{1}{2 N-2} \\
\frac{-1}{2 N} & \frac{1}{2 N-1} \frac{1}{2 N-2} & \frac{1}{2 N-3} & \cdots & 0
\end{array}\right]
$$

$=\left[\delta_{j k}^{(1)}\right]$,
$(2.47) I_{m}^{(2)}=\left[\begin{array}{ccccc}-\frac{\pi^{2}}{3} & \frac{2}{1^{2}} & -\frac{2}{2^{2}} & \ldots & \frac{-2}{(2 N)^{2}} \\ \frac{2}{1^{2}} & -\frac{\pi^{2}}{3} & \frac{2}{1^{2}} & \ldots & \frac{2}{(2 N-1)^{2}} \\ -\frac{2}{(2 N)^{2}} & \frac{2}{(2 N-1)^{2}} & -\frac{2}{(2 N-2)^{2}} & \cdots & -\frac{\pi^{2}}{3}\end{array}\right]=\left[\delta_{j k}^{(2)}\right]$.

With this notation Eqs. (2.39), (2.40) and (2.41) take the approximating form

$$
\begin{gathered}
\int_{\Gamma} r(x) f(x) \mathbf{S}_{m}(x) d x=h \mathbf{A}_{m}\left(\frac{r g}{\phi^{\prime}}\right) \mathbf{f}_{m} \\
\int_{\Gamma} r(x) f^{\prime}(x) \mathbf{S}_{m}(x) d x=-h\left[\mathbf{A}_{m}\left(\frac{(r g)^{\prime}}{\phi^{\prime}}\right)+\frac{1}{h} \mathbf{I}_{m}^{(1)} \mathbf{A}_{m}(r g)\right] \mathbf{f}_{m},
\end{gathered}
$$

$$
\begin{aligned}
& \int_{\Gamma} r(x) f^{\prime \prime}(x) \mathbf{S}_{m}(x) d x \\
& \quad=h\left[\mathbf{A}_{m}\left(\frac{(r g)^{\prime \prime}}{\phi^{\prime}}\right)+\frac{1}{h} \mathbf{I}_{m}^{(1)} \mathbf{A}_{m}\left(2(r g)^{\prime}+r g \phi^{\prime \prime} / \phi^{\prime}\right)+\frac{1}{h^{2}} \mathbf{I}_{m}^{(2)} \mathbf{A}_{m}\left(r g \phi^{\prime}\right)\right] \mathbf{f}_{m} .
\end{aligned}
$$

By [4, pp. 67-72] , the matrix $\mathbf{I}_{m}^{(1)}$ is simply related to a Toeplitz-type matrix, by considering the Fourier series expansion of $-i t$ on $(-\pi, \pi)$. The matrix $\mathrm{I}_{m}^{(2)}$ is a Toeplitz matrix, obtainable by considering the Fourier series expansion of $-t^{2}$ on $[-\pi, \pi]$. Thus, $\left[4\right.$, p. 65], the eigenvalues of $\mathrm{I}_{m}^{(1)}$ are $i \lambda_{k}^{(1)}, k=-N,-N+1, \ldots$, $N$, where $-\pi<\lambda_{k}^{(1)}<\pi$, while the eigenvalues of $\mathrm{I}_{m}^{(2)}$ are $-\lambda_{k}^{(2)}, k=-N,-N+1$, $\ldots, N$, where $0<\lambda_{k}^{(2)}<\pi^{2}$. Indeed, let $0<\lambda_{-N}^{(2)} \leqslant \lambda_{-N+1}^{(2)} \leqslant \cdots \leqslant \lambda_{N}^{(2)}<\pi^{2}$. Then by [4, pp. 64 and 67], since $x^{2} \geqslant 2-2 \cos x$ on [- $\left.-\pi, \pi\right]$, it follows that $\lambda_{-N}^{(2)}>2-2 \cos [\pi /(2 N+2)]=4 \sin ^{2}[\pi /(4 N+4)]$. That is, $\left|\lambda_{N}^{(2)} / \lambda_{-N}^{(2)}\right|$, the condition number of $\mathbf{I}_{m}^{(2)}$ is bounded by $\pi^{2} /\left\{4 \sin ^{2}[\pi /(4 N+4)]\right\} \sim(N+1)^{2}$. Summing up, we have 
THEOREM 2.12. (a) $\mathbf{I}_{m}^{(1)}$ is a skew-symmetric matrix having determinant zero. The eigenvalues $i \lambda_{k}^{(1)}$ of $\mathrm{I}_{m}^{(1)}$ satisfy the inequality $-\pi<\lambda_{k}^{(1)}<\pi, k=-N,-N+$ $1, \ldots, N$. (b) $\mathbf{I}_{m}^{(2)}$ is a negative definite matrix having eigenvalues $-\lambda_{k}^{(2)}$, where $4 \sin ^{2}[\pi /(4 N+4)]<\lambda_{k}^{(2)}<\pi^{2}$.

We close this section with a derivation of the formulas of Theorem 2.10 , for the case of the important intervals $[0,1],[-1,1],[0, \infty]$, and $[-\infty, \infty]$.

Example 1. $\Gamma=[0,1]$. In this case

$$
\left\{\begin{array}{l}
\phi(z)=\log \left(\frac{z}{1-z}\right), \quad \phi^{\prime}(z)=\frac{1}{z(1-z)} \\
D=\left\{z:\left|\arg \left(\frac{z}{1-z}\right)\right|<d\right\} .
\end{array}\right.
$$

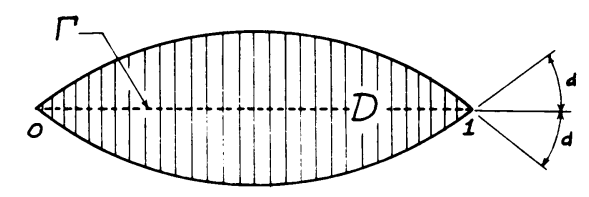

Figure 2.1. D of Example 1

Let us assume that the coefficients $r$ of a second order equation are analytic in $D$, and that the same is true of $r^{\prime}$ and $r^{\prime \prime}$. It is then convenient to take

$$
g(x)=\frac{1}{\phi^{\prime}(x)}=x(1-x)
$$

The conditions of Theorem 2.10 are satisfied if $f$ is analytic and bounded on $D$, and if on $[0,1],|f(x)| \leqslant C|x(1-x)|^{\alpha}$, where $C$ and $\alpha$ are positive constants. If $f$ does not vanish at 0 and 1 , we replace $f$ by $F$ in the differential equation, where

$$
F(x)=f(x)-a(1-x)-b x
$$

and where $a=f(0), b=f(1)$. The functions $S_{k}$ are

$$
\left\{S_{k}(x)\right\}_{k=-N}^{N}=\{x(1-x) S(k, h) \circ \phi(x)\}_{k=-N}^{N}
$$

To the basis functions $\left\{S_{k} / g\right\}$ it may be necessary to adjoin $1-x$ if $a$ is unknown, and $x$ if $b$ is unknown. Differentiating $g$ and $\phi^{\prime}$, we get

$$
g^{\prime}(x)=1-2 x, \quad g^{\prime \prime}(x)=-2, \quad \phi^{\prime \prime}(x)=-\frac{1-2 x}{x^{2}(1-x)^{2}}
$$

Hence, 


$$
\left\{\begin{array}{l}
(r g)(x)=x(1-x) r(x) ;\left(\frac{r g}{\phi^{\prime}}\right)(x)=x^{2}(1-x)^{2} r(x) \\
\left(\frac{(r g)^{\prime}}{\phi^{\prime}}\right)(x)=x(1-x)\left[x(1-x) r^{\prime}(x)+(1-2 x) r(x)\right] \\
\left(\frac{(r g)^{\prime \prime}}{\phi^{\prime}}\right)(x)=x(1-x)\left[x(1-x) r^{\prime \prime}(x)+2(1-2 x) r^{\prime}(x)-2 r(x)\right] \\
\left(\frac{2(r g)^{\prime} \phi^{\prime}+r g \phi^{\prime \prime}}{\phi^{\prime}}\right)(x)=2 x(1-x) r^{\prime}(x)+(1-2 x) r(x) \\
\left(r g \phi^{\prime}\right)(x)=r(x)
\end{array}\right.
$$

Hence, we get the approximations (2.48), in which $x_{k}=1 / 2+1 / 2 \tanh (k h / 2)$.

Example 2. $\Gamma=[-1,1]$. In this case

$$
\begin{aligned}
& \phi(z)=\log \left(\frac{1+z}{1-z}\right), \quad \phi^{\prime}(z)=\frac{2}{1-z^{2}}, \\
& D=\left\{z:\left|\arg \left(\frac{1+z}{1-z}\right)\right|<d\right\} .
\end{aligned}
$$

Under assumptions on $r$ similar to those of Example 1, we take

$$
g(x)=\frac{1}{\phi^{\prime}(x)}=\frac{1}{2}\left(1-x^{2}\right) .
$$

The conditions of Theorems 2.9 and 2.10 are satisfied if $f$ is analytic and bounded in $D$, and if on $(-1,1),|f(x)| \leqslant C\left(1-x^{2}\right)^{\alpha}$, where $C$ and $\alpha>0$. If $f$ does not vanish on -1 and 1 , we set $f=F+p$ in the differential equation, where

$$
p(x)=a \frac{1-x}{2}+b \frac{1+x}{2}
$$

and where $a=f(-1), b=f(1)$. The functions $S_{k}$ are

$$
\left\{S_{k}(x)\right\}_{k=-N}^{N}=\left\{\frac{1}{2}\left(1-x^{2}\right) S(k, h) \circ \phi(x)\right\}_{k=-N}^{N}
$$

to $\left\{S_{k} / g\right\}$ it may be necessary to adjoin $(1-x) / 2$ and/or $(1+x) / 2$ if $a$ and/or $b$ are unknown. Differentiating $g$ and $\phi^{\prime}$, we get

$$
g^{\prime}(x)=-x, \quad g^{\prime \prime}(x)=-1, \quad \phi^{\prime \prime}(x)=x\left(\frac{2}{1-x^{2}}\right)^{2},
$$

so that

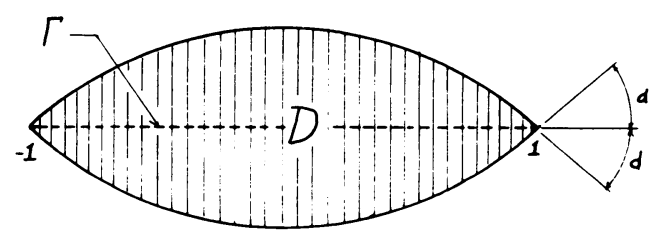

FIgure 2.2. D of Example 2 


$$
\left\{\begin{array}{l}
(r g)(x)=\frac{1}{2}\left(1-x^{2}\right) r(x) ; \quad\left(\frac{r g}{\phi^{\prime}}\right)(x)=\left(\frac{1-x^{2}}{2}\right)^{2} r(x) \\
\left(\frac{(r g)^{\prime}}{\phi^{\prime}}\right)(x)=\left(\frac{1-x^{2}}{2}\right)^{2} r^{\prime}(x)-x\left(\frac{1-x^{2}}{2}\right) r(x) \\
\left(\frac{(r g)^{\prime \prime}}{\phi^{\prime}}\right)(x)=\left(\frac{1-x^{2}}{2}\right)^{2} r^{\prime \prime}(x)-x\left(1-x^{2}\right) r^{\prime}(x)-\left(\frac{1-x^{2}}{2}\right) r(x) \\
\left(2(r g)^{\prime}+r g \phi^{\prime \prime} / \phi^{\prime}\right)(x)=\left(1-x^{2}\right) r^{\prime}(x)-x r(x) \\
\left(r g \phi^{\prime}\right)(x)=r(x)
\end{array}\right.
$$

Hence, with $x_{k}=\tanh (k h / 2)$, the approximations of Theorem 2.10 take the form (2.48).

Example 3. The case $\Gamma=[0, \infty]$. In this case

$$
\phi(z)=\log z, \quad \phi^{\prime}(z)=\frac{1}{z}, \quad D=\{z:|\arg z|<d\} .
$$

Suppose that the coefficients $r$ are analytic in $D$. If on $D$,

$$
|f(z)| \leqslant C|z|^{\alpha} /(1+|z|)^{2 \alpha},
$$

where $C, \alpha$ are positive then it is convenient to take $g(z)=z /(1+z)^{2}$, in order that the conditions of Theorems 2.9 and 2.10 are satisfied. However, if $|f(z)| \leqslant$ $C|z|^{\alpha} /(1+|z|)^{2+\alpha}$ in $D$, where $C$ and $\alpha$ are positive, then it is possible to choose a simpler form for $g$, and $S_{k}(x)$, namely

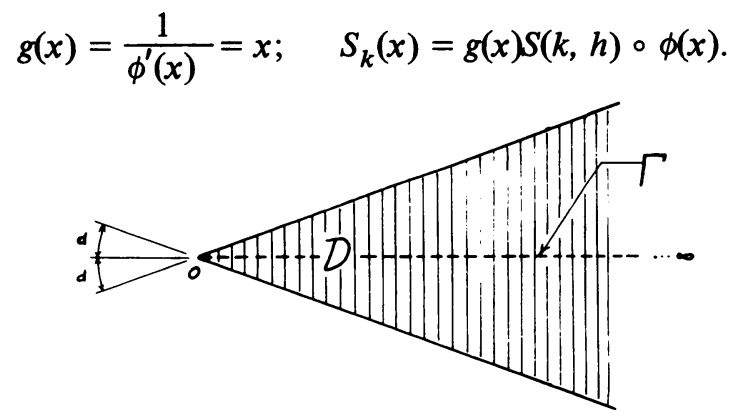

FIgure 2.3. D of Example 3

In this latter case

$$
\left\{\begin{array}{l}
(r g)(x)=x r(x) ; \quad\left(\frac{r g}{\phi^{\prime}}\right)(x)=x^{2} r(x) \\
\left(\frac{(r g)^{\prime}}{\phi^{\prime}}\right)(x)=x^{2} r^{\prime}(x)+x r(x) ; \quad\left(\frac{(r g)^{\prime \prime}}{\phi^{\prime}}\right)(x)=x^{2} r^{\prime \prime}(x)+2 x r^{\prime}(x) \\
\left(2(r g)^{\prime}+r g \phi^{\prime \prime} / \phi^{\prime}\right)(x)=2 x r^{\prime}(x)+r(x) \\
\left(r g \phi^{\prime}\right)(x)=r(x)
\end{array}\right.
$$


The approximations now take the form (2.48), in which $x_{k}=e^{k h}$.

If $f$ is merely bounded on $D$, and if $d=\lim _{(x \rightarrow \infty)} x^{2} f^{\prime}(x)$, then we replace $f$ by $F$ in the differential equation, where

$$
f(x)=F(x)+\frac{a}{1+x}+\frac{x b}{1+x}+\frac{x c}{(1+x)^{2}},
$$

where

$$
a=f(0), \quad b=f(\infty), \quad c=b-a-d .
$$

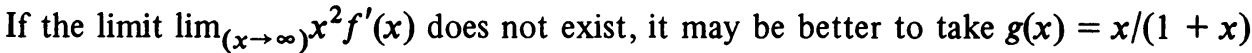
or $g(x)=x /(1+x)^{2}$, depending upon the problem.

Example 4. The case $\Gamma=[0, \infty]$. For some differential equation problems over $[0, \infty]$ it may not be possible to prove that the solution is analytic in a sector; rather it may be possible to prove analyticity only in the strip

$$
D=\{z:|\arg \sinh z|<d\} \text { (see Figure 2.4), } d \leqslant \pi / 2 .
$$

In this case it is convenient to take

$$
\left\{\begin{array}{l}
w=\phi(z)=\log \sinh z \\
\phi^{\prime}(z)=\sqrt{1+e^{-2 w}}
\end{array}\right.
$$

Best results are achieved for solutions $f$ which are analytic in $D$ and which satisfy $|f(x)| \leqslant C x^{\alpha} e^{-\alpha x}$ on $[0, \infty]$, where $\alpha>0$. In this case

$$
\left\{\begin{array}{l}
g(x)=\frac{1}{\phi^{\prime}(x)}=\frac{1}{\sqrt{1+e^{-2 w}}} \\
S_{k}(x)=g(x) S(k, h) \circ \phi(x),
\end{array}\right.
$$

and the solution is approximated as follows:

$$
f(x) \cong \sum_{k=-N}^{N} f_{k} S(k, h) \circ \phi(x),
$$

where $f_{k} \cong f\left(x_{k}\right)$ and $x_{k}=\log \left[e^{k h}+\sqrt{1+e^{2 k h}}\right]$.

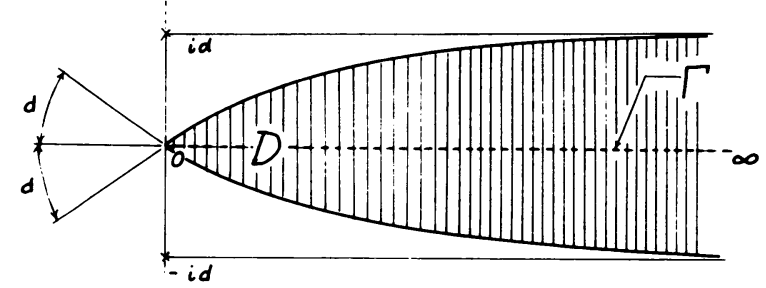

Figure 2.4. D of Example 4 
Then

$$
\left\{\begin{array}{l}
(r g)(x)=\frac{1}{\sqrt{1+e^{-2 w}}} r(x) ; \quad\left(\frac{r g}{\phi^{\prime}}\right)(x)=\frac{1}{1+e^{-2 w}} r(x), \\
\left(\frac{(r g)^{\prime}}{\phi^{\prime}}\right)(x)=\frac{1}{1+e^{-2 w}} r^{\prime}(x)+\frac{e^{-2 w}}{\left(1+e^{-2 w}\right)^{3 / 2}} r(x), \\
\frac{(r g)^{\prime \prime}}{\phi^{\prime}}=\frac{1}{1+e^{-2 w}} r^{\prime \prime}(x)+\frac{2 e^{-2 w}}{\left(1+e^{-2 w}\right)^{3 / 2}} r^{\prime}(x)-\frac{2 e^{-2 w}}{\left(1+e^{-2 w}\right)^{2}} r(x), \\
\left(2(r g)^{\prime}+r g \phi^{\prime \prime} / \phi^{\prime}\right)(\dot{x})=\frac{2}{\sqrt{1+e^{-2 w}} r^{\prime}(x)+\frac{e^{-2 w}}{1+e^{-2 w}} r(x),} \\
\left(r g \phi^{\prime}\right)(\dot{x})=r(x),
\end{array}\right.
$$

in which we may substitute $x_{k}$ for $x$ and $k h$ for $w$.

If $f$ is bounded but does not vanish at 0 and/or $\infty$, we can replace $f$ by $F$ in the differential equations, where

$$
F(x)=f(x)-f(0) e^{-x}-f(\infty)\left(1-e^{-x}\right)
$$

Example 5. The case $\Gamma=[-\infty, \infty]$. In this case

$$
\phi(z)=z, \quad \phi^{\prime}(z)=1,
$$

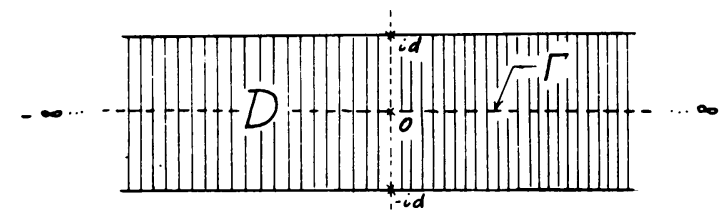

Figure 2.5. D of Example 5

and $D=D_{d}^{\prime}$ (see Eq. (2.12)). If the coefficients $r$ of the differential equation are analytic in $D_{d}^{\prime}$, and if $f \in B\left(D^{\prime}\right)$, we simply take

$$
g(x)=1, \quad\left\{S_{k}(x)\right\}_{k=-N}^{N}=\{S(k, h)(x)\}_{k=-N}^{N}
$$

in order that the conditions of Theorem 2.9 become satisfied, and provided that $f$ vanishes at $\pm \infty$. The conditions of Theorem 2.10 also become satisfied if $|f(x)| \leqslant$ $C e^{-\alpha|x|}$ on $\Gamma$. Then $x_{k}=k h$, and 


$$
\left\{\begin{array}{l}
(r g)(x)=r(x), \quad\left(\frac{r g}{\phi^{\prime}}\right)(x)=r(x), \quad\left(\frac{(r g)^{\prime}}{\phi^{\prime}}\right)(x)=r^{\prime}(x), \\
\left(\frac{(r g)^{\prime \prime}}{\phi^{\prime}}\right)(x)=r^{\prime \prime}(x), \quad\left(2(r g)^{\prime}+r g \phi^{\prime \prime} / \phi^{\prime}\right)(x)=2 r^{\prime}(x), \\
\left(r g \phi^{\prime}\right)(x)=r(x) .
\end{array}\right.
$$

The approximating equations again take the form (2.48).

If $f$ does not vanish at $\pm \infty$, we replace $f$ by $F$, where

$$
F(x)=f(x)-\frac{1}{e^{c x}+e^{-c x}}\left[e^{-c x} f(-\infty)+e^{c x} f(\infty)\right]
$$

and where $0<c<\pi /(2 d)$.

3. Examples of Applications. In this section we shall illustrate the application of the formulas developed in Section 2, on the solution of some simple ordinary and partial differential equations.

Example 1. Consider the simple problem

$$
f_{x x}(x)=-2, \quad 0<x<1 ; \quad f(0)=f(1)=0 .
$$

This has the solution $f(x)=x(1-x)$. By taking $r(x)=1$ in (2.54) and combining with (2.48), we arrive at the system of equations

$$
\begin{aligned}
h\left[2 \mathbf{A}_{m}(x(1-x))\right. & \left.+\frac{1}{h} \mathbf{I}_{m}^{(1)} \mathbf{A}_{m}(1-2 x)+\frac{1}{h^{2}} \mathbf{I}_{m}^{(2)}\right] \mathbf{f}_{m} \\
& =-2 h \mathbf{A}_{m}\left(x^{2}(1-x)^{2}\right) \mathbf{e}
\end{aligned}
$$

where $\mathbf{e}=(1,1, \ldots, 1)^{T}, T$ denoting the transpose. Solving this system for the case $N=16, h=.75 / N^{1 / 2}, x_{k}=1 / 2+1 / 2 \tanh (k h / 2)$, we get an approximate solution

$$
f(x) \cong \sum_{k=-16}^{16} f_{k} S(k, h) \circ \phi(x) \quad(\phi(x)=\log [x /(1-x)])
$$

which is accurate to 5 decimals on $[0,1]$. Similar accuracy obtains if the -2 in (3.1) is replaced by $-2 x^{\alpha-2}(1-x)^{\beta-2}, \alpha, \beta>0$.

Example 2. $f^{\prime \prime}=f-f^{3} / x^{3}, f(0)=f(\infty)=0$. This problem was solved by different procedures in [1] and [6]. By taking $x_{k}=e^{k h}$ and combining (2.63) and (2.48), we get the approximating system

$$
\left[\mathbf{I}_{m}^{(1)}+\frac{1}{h} \mathbf{I}_{m}^{(2)}\right] \mathbf{f}_{m}=h \mathbf{A}_{m}\left(x^{2}\right)\left[\mathbf{f}_{m}-\mathbf{e}_{m}\right],
$$

where $\mathrm{e}_{m}$ denotes the vector $\left[x_{-N}^{-2} f_{-N}^{3}, x_{-N+1}^{-2} f_{-N+1}^{3}, \ldots, x_{N}^{-2} f_{N}^{3}\right]^{T}$. The solution of (3.3) involves the solution of a system of nonlinear equations. By taking $h=$ 
$.5 / N^{1 / 2}, N=16$ we get an approximate solution ${ }^{3}$

$$
f(x) \cong \sum_{k=-16}^{16} f_{k} S(k, h) \circ \phi(x) \quad(\phi(x)=\log x)
$$

which is accurate to 5 decimals on $[0, \infty]$.

Example 3.

$$
\left\{\begin{array}{l}
u_{x x}=u_{t}, \quad 0<x<1, t>0 \\
u(x, 0)=\sin \pi x, u(0, t)=u(1, t)=0
\end{array}\right.
$$

In order to get zero boundary conditions, we set

$$
u=v+\sin (\pi x) e^{-4 t} \text {. }
$$

This yields the problem

$$
\left\{\begin{array}{l}
v_{x x}-v_{t}=\left(\pi^{2}-4\right) \sin (\pi x) e^{-4 t}, \quad 0<x<1, t>0 \\
v(x, 0)=0, \quad v(0, t)=v(1, t)=0 .
\end{array}\right.
$$

We solve this by taking our approximating basis functions to be

$$
\left\{\begin{array}{l}
S_{k}(x)=x(1-x) S(k, h) \circ \phi(x), \quad \phi(x)=\log [x /(1-x)] \\
S_{l}^{*}(t)=t S(l, s) \circ \phi^{*}(t), \quad \phi^{*}(t)=\log t
\end{array}\right.
$$

The problem (3.6) may now readily be reduced to a matrix problem, by proceeding as for (3.2) above. Setting

$(3.8) \mathrm{V}=\left[\begin{array}{crrr}v_{-N, N} & v_{-N,-N+1} & \cdots & v_{-N N} \\ v_{-N+1,-N} & v_{-N+1,-N+1} & \ldots & v_{-N+1, N} \\ & \cdot & \cdot & \\ v_{N,-N} & v_{N,-N+1} & \cdots & v_{N N}\end{array}\right]$,

$$
\mathbf{B}=-2 h \mathbf{A}_{m}(x(1-x))+\mathbf{I}_{m}^{(1)} \mathbf{A}_{m}(1-2 x)+\frac{1}{h} \mathbf{I}_{m}^{(2)} \quad\left(x_{k}=\frac{1}{2}+\frac{1}{2} \tanh \frac{k h}{2}\right),
$$

\footnotetext{
${ }^{3}$ The solution $f$ satisfies $f(x) \sim A e^{-x}$ as $x \rightarrow \infty$, and consequently, it may be necessary, on some computers, to replace $\Sigma_{-N}^{N}$ by $\Sigma_{-N}$, where $M<<N$, in order to avoid underflow. The method of Example 4, Section 2 avoids this difficulty and produces a more accurate solution to this problem.
} 


$$
\begin{aligned}
& \mathbf{C}=\mathbf{A}_{m}(t)\left[s \mathbf{I}_{m}^{(0)}-\mathbf{I}_{m}^{(1)}\right] \quad\left(\mathbf{I}_{m}^{(0)}=\text { unit matrix, } t_{k}=e^{k s}\right) \\
& \mathbf{D}=h \mathbf{A}_{m}\left(x^{2}(1-x)^{2}\right) \quad\left(x_{k}=1 / 2+1 / 2 \tanh (k h / 2)\right), \\
& \mathbf{E}=\left[\begin{array}{crcc}
F_{-N,-N} & F_{-N,-N+1} & \cdots & F_{-N, N} \\
F_{-N+1,-N} & F_{-N+1,-N+1} & \cdots & F_{-N+1, N} \\
& . & . & \\
F_{N,-N} & F_{N,-N+1} & \cdots & F_{N N}
\end{array}\right] \text {, }
\end{aligned}
$$

where

$$
F_{k l}=\left(\pi^{2}-4\right) \sin \left(\pi x_{k}\right) e^{-4 t} l
$$

we arrive at the matrix system

$$
\mathbf{D}^{-1} \mathbf{B V}+\mathrm{VCE}^{-1}=\mathbf{F} \text {. }
$$

Equation (3.14) may be solved by diagonalizing $\mathbf{D}^{-1} \mathbf{B}$ and $\mathbf{C E} \mathbf{E}^{-1}$. If $\lambda_{-N}$, $\lambda_{-N+1}, \ldots, \lambda_{N}$ and $\mu_{-N}, \mu_{-N+1}, \ldots, \mu_{N}$ denote the eigenvalues of $\mathbf{D}^{-1} \mathbf{B}$ and and $\mathbf{C E}^{-1}$, respectively, obtained by taking $X^{-1} \mathbf{D}^{-1} \mathbf{B X}$ and $Z_{C E}^{-1} Z^{-1}$ via e.g. the method of Golub and Reinsch [3], and if $G=\left[g_{k l}\right]=X^{-1} F Z^{-1}, Y=\left[y_{k l}\right]=$ $\mathbf{X}^{-1} \mathbf{V Z}^{-1}$ then $y_{k l}=g_{k l} /\left(\lambda_{k}+\mu_{l}\right)$, and $\mathbf{V}=\mathbf{X Y Z}$.

By taking $h=.75 / N^{1 / 2}, N=.5 / N^{1 / 2}, N=16$, we get an approximation

$$
u(x, t) \cong e^{-4 t} \sin \pi x+\sum_{k, l=-16}^{16} v_{k l} S(k, h) \circ \phi(x) S(l, h) \circ \phi^{*}(t),
$$

which is accurate to 4 decimals on $[0,1] \times[0, \infty] .4$

Example 4.

$$
\begin{gathered}
u_{x x}+u_{y y}=-1, \quad(x, y) \in S \equiv[0,1] \times[0,1] \\
u=0 \text { on } \partial S .
\end{gathered}
$$

Letting $B$ and $D$ be defined as in (3.9) and (3.11) we now get the approximating matrix system

$$
\mathrm{D}^{-1} \mathrm{BU}+\mathrm{U}\left(\mathrm{D}^{-1} \mathrm{~B}\right)^{T}=-\mathrm{H}
$$

where $\mathbf{U}=\left[u_{k l}\right], \mathbf{H}=\left[h_{k l}\right], h_{k l}=1$. This may now be readily solved via the

${ }^{4}$ The choice of $S_{l}^{*}(t)$ given in Example 4 of Section 2 would have yielded 5 decimal accuracy. 
diagonalization of $\mathbf{D}^{-1} \mathbf{B}$. By taking $N=16, h=.75 / N^{1 / 2}$ we get an approximate solution

$$
u(x, y) \cong \sum_{k, l=-16}^{16} u_{k l} S(k, h) \circ \phi(x) S(l, h) \circ \phi(y),
$$

which is accurate to 5 decimals on $S$.

4. Error Analysis. For sake of simplicity, we shall restrict ourselves to the simpler case of the second order problem

$$
u^{\prime \prime}+f(x, u)=0, \quad u(0)=u(1)=0 .
$$

The analysis for the case of other ordinary or partial differential equations is somewhat more complicated, but may be carried out similarly.

Throughout this section $\alpha, C_{1}, C_{2}, \ldots, C_{16}$ denote positive constants, and $h=\left[\pi d /(\alpha N)^{1 / 2}\right]$.

In the notation of the previous sections, we take $\phi(z)=\log [z /(1-z)]$, and we take the domain of analyticity to be $D=\{z:|\arg [z /(1-z)]|<d\}$. We shall assume that (4.1) has a (locally) unique solution $u_{0}$ which is analytic and bounded in $D$ and which satisfies the inequality

$$
\left|u_{0}(x)\right| \leqslant C_{1} x^{\alpha}(1-x)^{\alpha}, \quad 0 \leqslant x \leqslant 1
$$

Definition 4.1. Let $M(d, \alpha)$ denote the family of all functions $v$ that are analytic in $D$, such that

$$
\left\{\begin{array}{l}
v(0)=v(1)=0 \\
g v^{\prime \prime} \in B(D), \quad\left|g(x) v^{\prime \prime}(x)\right| \leqslant C_{2} x^{\alpha-1}(1-x)^{\alpha-1} \quad \text { on }(0,1) \\
g f(\cdot, v) \in B(D), \quad|g(x) f(x, v(x))| \leqslant C_{3} x^{\alpha-1}(1-x)^{\alpha-1} \quad \text { on }(0,1)
\end{array}\right.
$$

where

$$
g(x)=x(1-x)
$$

We shall also assume that the solution of the Fréchet derivative problem

$$
\theta^{\prime \prime}(x)+f_{u}(x, u(x)) \theta(x)=w(x), \quad \theta(0)=\theta(1)=0,
$$

satisfies

$$
|\theta(x)| \leqslant C_{4}\left\|A^{-1} w\right\|
$$

for all $u \in M(d, \alpha)$ such that $\left\|u-u_{0}\right\| \leqslant \epsilon$, where $\|\cdot\|$ is defined by

$$
\|f\|=\sup _{x \in(0,1)}|f(x)|,
$$


where

$$
\left(A^{-1} f\right)(x)=-\int_{0}^{1} G(x, t) f(t) d t,
$$

and where for any $x \in[0,1]$,

$$
G(x, t)= \begin{cases}(1-x) t & \text { if } 0 \leqslant t \leqslant x \\ x(1-t) & \text { if } x \leqslant t \leqslant 1\end{cases}
$$

Moreover, we shall assume that if $\left\|u-u_{0}\right\| \leqslant \epsilon$, then

$$
\left\|\left\{A^{-1} f(t, u(t))\right\}\right\| \leqslant C_{5} .
$$

Let us assume that we have found an approximate solution

$$
u_{m}(x)=\sum_{k=-N}^{N} u_{k} S(k, h) \circ \phi(x) \quad(m=2 N+1)
$$

by the method of the previous sections, and let us set

$$
\theta_{m}=u_{m}-u_{0}
$$

Then

$$
\theta_{m}^{\prime \prime}(x)+f_{u}(x, \bar{u}(x)) \theta_{m}(x)=u_{m}^{\prime \prime}(x)+f\left(x, u_{m}(x)\right)
$$

for some $\bar{u}$ between $u_{0}$ and $u_{m}$; and therefore, by (4.5) and (4.6),

$$
\left|\theta_{m}(x)\right| \leqslant C_{4}\left\|u_{m}+A^{-1} f\left(; u_{m}\right)\right\| \text {. }
$$

Now by Theorem 2.1, we find, by taking $S_{k}(x)=g(x) S(k, h) \circ \phi(x), x_{k}=$ $1 / 2+1 / 2 \tanh (k h / 2)$, that

$$
\int_{0}^{1}\left[v^{\prime \prime}(x)+f(x, v(x))\right] S_{k}(x) d x \cong h \frac{g\left(x_{k}\right)}{\phi^{\prime}\left(x_{k}\right)}\left[v^{\prime \prime}\left(x_{k}\right)+f\left(x_{k}, v\left(x_{k}\right)\right)\right]
$$

and

$$
\int_{0}^{1} v^{\prime \prime}(x) S_{k}(x) d x
$$

$$
\cong h \sum_{j=-N}^{N} v\left(x_{j}\right)\left[\frac{g^{\prime \prime}\left(x_{j}\right)}{\phi^{\prime}\left(x_{j}\right)} \delta_{k j}^{(0)}+\left\{2 g^{\prime}\left(x_{j}\right)+g\left(x_{j}\right) \phi^{\prime \prime}\left(x_{j}\right) / \phi^{\prime}(x)\right\} \frac{\delta_{k j}^{(1)}}{h}\right.
$$

$$
\left.+g\left(x_{j}\right) \phi^{\prime}\left(x_{j}\right) \frac{\delta_{k j}^{(2)}}{h^{2}}\right]
$$

in which the error of either term on the right-hand side of (4.15) is bounded by $C_{6} N^{-1 / 2} e^{-(\pi d \alpha N)^{1 / 2}}$, and the error of the right-hand side of $(4.16)$ is bounded by $C_{7} N^{1 / 2} e^{-(\pi d \alpha N)^{1 / 2}}$. By our process of solution, the numbers $u_{k}$ in (4.11) are deter- 
mined such that

$$
\begin{aligned}
h \sum_{j=-N}^{N} u_{j}\left[\frac{g_{j}^{\prime \prime}}{\phi_{j}^{\prime}} \delta_{k j}^{(0)}+\left\{2 g_{j}^{\prime}+g_{j} \phi_{j}^{\prime \prime} / \phi_{j}^{\prime}\right\} \frac{\delta_{j k}^{(1)}}{h}+g_{j} \phi_{j}^{\prime} \frac{\delta_{k j}^{(2)}}{h^{2}}\right] \\
+h \frac{g_{k}}{\phi_{k}^{\prime}} f\left(x_{k}, u_{k}\right)=0, \quad k=-N,-N+1, \ldots, N
\end{aligned}
$$

THEOREM 4.2. Let the numbers $u_{k}(k=-N,-N+1, \ldots, N)$ be determined by (4.17), and let $u_{m}(x)$ be defined as in (4.11). Then

$$
\left|u_{m}(x)-u_{0}(x)\right| \leqslant C_{15} N^{3 / 2} e^{-(\pi d \alpha N)^{1 / 2}}, \quad 0 \leqslant x \leqslant 1,
$$

where $u_{0}$ is the solution of (4.1).

Proof. In view of the errors in the approximations (4.15) and (4.16), the solution of (4.17) is equivalent to finding a function $v \in M(d, \alpha)$, such that

$$
\frac{g\left(x_{k}\right)}{\phi^{\prime}\left(x_{k}\right)}\left[v^{\prime \prime}\left(x_{k}\right)+f\left(x_{k}, v\left(x_{k}\right)\right)\right]=\frac{\epsilon_{k}}{h}, \quad k=-N,-N+1, \ldots, N,
$$

where $v\left(x_{k}\right)=u_{k}$, and where

$$
\left|\epsilon_{k}\right| \leqslant C_{8} N^{1 / 2} e^{-(\pi d \alpha N)^{1 / 2}}, \quad k=-N,-N+1, \ldots, N .
$$

Since $v \in M(d, \alpha)$, it follows, for any $t \in(0,1)$, that

$$
\begin{gathered}
\frac{g(t)}{\phi^{\prime}(t)}\left[v^{\prime \prime}(t)+f(t, v(t))\right]-\sum_{k=-\infty}^{\infty} \frac{g\left(x_{k}\right)}{\phi^{\prime}\left(x_{k}\right)}\left[v^{\prime \prime}\left(x_{k}\right)+f\left(x_{k}, v\left(x_{k}\right)\right)\right] S(k, h) \circ \phi(t) \\
=\frac{\sin [\pi \phi(t) / h]}{2 \pi i} \int_{\partial v} \frac{g(z)\left[v^{\prime \prime}(z)+f(z, v(z))\right] d z}{[\phi(z)-\phi(t)] \sin [\pi \phi(z) / h]} .
\end{gathered}
$$

By multiplying (4.21) by $\phi^{\prime}(t)^{2}$, taking $A^{-1}$ of each side, and noting that $g(t) \phi^{\prime}(t)=1$, we get

$$
v(x)+\left\{A^{-1} f(t, v(t))\right\}(x)-\sum_{k=-\infty}^{\infty} \frac{g_{k}}{\phi_{k}^{\prime}}\left[v_{k}^{\prime \prime}+f\left(x_{k}, v_{k}\right)\right] A^{-1}\left\{\phi^{\prime}(t)^{2} S(k, h) \circ \phi(t)\right\}(x)
$$

$$
=A^{-1}\left\{\frac{\phi^{\prime}(t)^{2} \sin [\pi \phi(t) / h]}{2 \pi i} \int_{\partial D} \frac{g(z)\left[v^{\prime \prime}(z)+f(z, v(z))\right] d z}{[\phi(z)-\phi(t)] \sin [\pi \phi(z) / h]}\right\}
$$

Since $\phi^{\prime}(t)=1 /[t(1-t)]$, it follows, by taking $t=[1+\tanh (u / 2)] / 2, x=$ $[1+\tanh (w / 2)] / 2$, and using (4.8) and (4.9), that

$$
\begin{aligned}
I_{1}(h, x) \equiv & A^{-1}\left\{\phi^{\prime}(t)^{2} \operatorname{sinc}[\{\phi(t)-k h\} / h]\right\}(x) \\
= & -\int_{-\infty}^{w} \frac{1-\tanh (w / 2)}{1-\tanh (u / 2)} \operatorname{sinc}\left[\frac{u-k h}{h}\right] d u \\
& -\int_{w}^{\infty} \frac{1+\tanh (w / 2)}{1+\tanh (u / 2)} \operatorname{sinc}\left[\frac{u-k h}{h}\right] d u .
\end{aligned}
$$


On the interval $[-\infty, w]$, the function $[1-\tanh (w / 2)] /[1-\tanh (u / 2)]$ increases monotonically from $[1-\tanh (w / 2)] / 2$ to 1 , while on $[w, \infty]$ the function $[1+\tanh (w / 2)] /[1+\tanh (u / 2)]$ decreases monotonically from 1 to $[1+\tanh (w / 2)] / 2$. For this reason, it may be shown by a somewhat lengthy, but simple argument, that

$$
\left|I_{1}(h, x)\right| \leqslant 4 \pi h \text {. }
$$

Similarly, if $x \in[0,1]$ and $z \in \partial D$, we can show that

$$
\left|I_{2}(h, x)\right| \equiv\left|A^{-1}\left\{\frac{\phi^{\prime}(t)^{2} \sin [\pi \phi(t) / h]}{2 \pi i[\phi(z)-\phi(t)]}\right\}(x)\right| \leqslant \frac{2 h}{d}
$$

since $\operatorname{Im} \phi(z)= \pm d$.

By means of (4.19), (4.22) and (4.25), Eq. (4.21) now yields

$$
\left|v(x)+\left\{A^{-1} f(t, v(t))\right\}(x)\right|
$$

$$
\begin{aligned}
& \leqslant\left|I_{1}(h, x)\right| \sum_{k=-N}^{N} \frac{\left|\epsilon_{k}\right|}{h}+\left|I_{1}(h, x)\right| \sum_{|k|>N} \frac{g_{k}}{\phi_{k}^{\prime}}\left|\left[v_{k}^{\prime \prime}+f\left(x_{k}, v_{k}\right)\right]\right| \\
& \quad+\left|I_{2}(h, x)\right| \int_{\partial D}\left|\frac{g(z)\left[v^{\prime \prime}(z)+f(z, v(z))\right]}{\sin [\pi \phi(z) / h]} d z\right| .
\end{aligned}
$$

Using the bounds given in (4.20) and (4.24), we bound the first sum on the righthand side of (4.26) by $C_{9} N^{3 / 2} e^{-(\pi d \alpha N)^{1 / 2}}$; using (4.3) and (4.24), and recalling that $x_{k}=1 / 2+1 / 2 \tanh (k h / 2)$, we bound the second sum on the right-hand side of (4.26) by $C_{10} e^{-(\pi d \alpha N)^{1 / 2}}$; and using (4.25) and the fact that $|\sin [\pi \phi(z) / h]| \geqslant \sinh [\pi d / h]$ if $z \in \partial D$, we bound the integral term on the right-hand side of (4.24) by

$$
\left|v(x)+\left\{A^{-1} f(t, v(t))\right\}(x)\right| \leqslant C_{12} N^{3 / 2} e^{-(\pi d \alpha N)^{1 / 2}} .
$$

Hence, for all $x \in[0,1]$,

$$
\left|v(x)+\left\{A^{-1} f(t, v(t))\right\}(x)\right| \leqslant C_{12} N^{3 / 2} e^{-(\pi d \alpha N)^{1 / 2}} .
$$

Since $v \in M(d, \alpha)$, it follows from the first and second of (4.3) that

$$
|v(x)| \leqslant C_{13} x^{\alpha}(1-x)^{\alpha}, \quad 0 \leqslant x \leqslant 1 .
$$

Furthermore, since $v \in M(d, \alpha)$, and since $u_{m}$ and $v$ coincide at $x_{-N}, x_{-N+1}, \ldots$, $x_{N}$, it follows that $[13$, Theorem 8.2] for all $x \in[0,1]$,

$$
\left|u_{m}(x)-v(x)\right| \leqslant C_{14} N^{1 / 2} e^{-(\pi d \alpha N)^{1 / 2}} .
$$

In view of (4.5), (4.6) and (4.10), it now follows that for all $x \in[0,1]$, 


$$
\begin{aligned}
\mid u_{m}(x)- & \left\{A^{-1} f\left(t, u_{m}(t)\right)\right\}(x) \mid \\
& \leqslant\left|v(x)+\left\{A^{-1} f(t, v(t))\right\}(x)\right|+C_{5}\left|u_{m}(x)-v(x)\right| .
\end{aligned}
$$

By (4.14), (4.27), (4.29) and (4.30), it thus follows that for all $x \in[0,1]$

$$
\left|\theta_{m}(x)\right|=\left|u_{m}(x)-u_{0}(x)\right| \leqslant C_{15} N^{3 / 2} e^{-(\pi d \alpha N)^{1 / 2}} .
$$

This completes the proof of Theorem 4.2.

Similarly, it may be shown that when using $n=(2 N+1)^{2}$ points to obtain an approximate solution of a partial differential equation, such as (3.15), the error is bounded by $C_{16} N^{3 / 2} e^{-\gamma N^{1 / 2}} \leqslant 5 C_{16} n^{3 / 4} e^{-\gamma n^{1 / 4}}$. Indeed, for the case of (3.15), we may take $C_{16}=1$ and $\gamma=\pi^{2}$.

Acknowledgements. The author is grateful to A. Adler and J. Varah at the University of British Columbia for valuable discussions involving Theorem 2.12 of this paper. The author also wishes to thank Bob Burke of the University of Utah for carrying out the computation in Section 3.

Department of Mathematics

University of British Columbia

Vancouver, B. C., Canada

1. J. CHAUVETTE \& F. STENGER, "The approximate solution of the nonlinear equation $\Delta u=u-u^{3}, " J$. Math. Anal. Appl., v. 51, 1975, pp. 229-242.

2. Y. H. CHIU, An Integral Equation Method of Solution of $\Delta u=k^{2} u, \mathrm{Ph}$. D. thesis, University of Utah, 1976.

3. G. GOLUB \& C. REINSCH, "Singular value decomposition and least squares solution," Numer. Math., v. 44, 1970, pp. 402-420.

4. U. GRENANDER \& G. SZEGÖ, Toeplitz Forms and Their Applications, Univ. of Calif. Press, 1958.

5. R. V. L. HARTLEY, "The transmission of information," Bell System Tech. J., v. 7, 1928 , pp. $535-560$.

6. L. LUNDIN, A Cardinal Function Method of Solution of $\Delta u=u-u^{3}$, Ph. D. thesis, University of Utah, 1975.

7. L. LUNDIN \& F. STENGER, "Cardinal-type approximations of a function and its derivatives," SIAM J. Math. Anal. (To appear.)

8. J. MCNAMEE, F. STENGER \& E. L. WHITTNEY, "Whittaker's cardinal function in retrospect," Math. Comp., v. 25, 1971, pp. 141-154.

9. H. NYQUIST, "Certain topics in telegraph transmission theory," Trans. Amer. Inst. Elec. Engrg., v. 47, 1928, pp. 617-644.

10. W. PETRICK, J. SCHWING \& F. STENGER, "An algorithm for the electromagnetic scattering due to an axially symmetric body with an impedance boundary condition," J. Math. Anal. Appl. (To appear.)

11. J. SCHWING, Eigensolutions of Potential Theory Problems in $R^{3}, \mathrm{Ph}$. D. thesis, University of Utah, 1976.

12. C. E. SHANNON, "A mathematical theory of communication,"Bell System Tech. J., v. 27, 1948, pp. 379-423, 623-656.

13. F. STENGER, "Approximations via Whittaker's cardinal function," J. Approximation Theory, v. 17, 1976, pp. 222-240.

14. F. STENGER, "Kronecker product extension of linear operators," SIAM J. Numer. Anal., v. 5, 1968, pp. 422-435.

15. F. STENGER, "Optimal convergence of minimum-norm approximations in $H_{p}$," Numer. Math., v. 29, 1978, pp. 345-362.

16. E. T. WHITTAKER, "On the functions which are represented by the expansions of the interpolation theory," Proc. Roy. Soc. Edinburgh, v. 35, 1915, pp. 181-194.

17. J. M. WHITTAKER, Interpolatory Function Theory, Cambridge, London, 1935. 